\title{
Comparing the Efficacy of Herbal and Non-herbal Toothpastes in Controlling Plaque and Gingivitis: A Review
}

\author{
S Nivethaprashanthi ${ }^{1}, \mathrm{R} \mathrm{Kavya}^{2}, \mathrm{R}$ Priyanga $^{3}, \mathrm{~N} \mathrm{Vezhavendhan}^{4}$
}

\begin{abstract}
Background and aim: Dental plaque, a well-organized biofilm, is the established cause for gingivitis. Chronic gingivitis is the second most common disease of oral cavity which may progress to periodontitis. A mechanical plaque control method along with dentifrices is the most recommended method for plaque removal and controlling gingivitis. The aim of this review is to estimate and compare the effectiveness of herbal and non-herbal toothpastes in controlling plaque and gingivitis.

Materials and methods: The primary focus of this study was to compare randomized control trials and clinical trials which used herbal toothpaste with non-herbal toothpaste in prevention of plaque and gingivitis. Clinical trials without randomization and other experimental studies were also included. The outcome measures were improvement in duration, objective and subjective difference in plaque accumulation, and gingivitis. Results: Randomized control trial and nonrandomized control trial has shown a remarkable reduction in plaque and gingival scores on an average duration of using herbal toothpaste. Studies (five randomized control trials and four clinical trials) state statistically equal reduction in plaque and gingival scores on using herbal and non-herbal toothpastes. No reports of adverse reactions (bad taste and odor, hard to accept, and allergic reactions) on using herbal toothpaste are evident.

Conclusion: Nowadays, there is an increased interest on using herbal products among people. Herbal toothpastes are as effective as nonherbal toothpastes in controlling plaque and gingivitis. There are also no adverse reactions on using herbal toothpaste and can be used as an alternative to conventional (non-herbal) toothpaste. Henceforth, it depends upon the preference of people to choose natural (herbal) or conventional (non-herbal) toothpaste.

Keywords: Gingival disease, Herbal toothpaste, Non-herbal toothpaste.

Journal of Scientific Dentistry (2020): 10.5005/jp-journals-10083-0920
\end{abstract}

\section{INTRODUCTION}

Systematically arranged biofilm, the dental plaque, is the causative factor of chronic gingivitis, one among the most common oral diseases. Chronic gingivitis if left untreated can progress to periodontitis, which further leads to loss of teeth. ${ }^{1}$ Gingivitis is the result of interactions between inflammatory cells and microorganisms found in dental plaque and gums, ${ }^{2}$ and numerous studies have proved that gingivitis is closely related to a number of systemic diseases. Hence, preventing and eliminating inflammation of the gingival region is crucial for maintaining a good oral health. ${ }^{3}$ Various studies conducted throughout the world proved that the incidence and prevalence of periodontal diseases are high, and dental plaque is virtually associated with it. Clinical studies related to the removal of plaque and remission of gingivitis had been accepted as support for the definite relationship between plaque and gingivitis and for the belief that it is a significant factor in the maintenance of gingival health. ${ }^{4}$

Dental plaque is composed of complex microorganisms in an organized manner, found in both the hard and soft tissue structures of the oral cavity. Dental plaque is one among the many causative factors for dental caries, gingivitis, and periodontitis. The second most common oral disease is the dental plaque-induced gingivitis, the first being dental caries. Dental calculus is a calcified structure (hardened plaque) which is yellow or brown in color and hard in consistency that cannot be removed by usual toothbrush while brushing. It requires specialized instruments, the scalars for removing it. ${ }^{5}$ Dental plaque can be mechanically removed by toothbrushing habit and is an effective method for preventing the oral diseases in which the dental plaque has a major contribution.
${ }^{1-4}$ Department of Oral and Maxillofacial Pathology and Oral Microbiology, Indira Gandhi Institute of Dental Sciences, Sri Balaji Vidyapeeth, Puducherry, India

Corresponding Author: S Nivethaprashanthi, Department of Oral and Maxillofacial Pathology and Oral Microbiology, Indira Gandhi Institute of Dental Sciences, Sri Balaji Vidyapeeth, Puducherry, India, Phone: +91 9500559944, e-mail: nivethaswaminathan@gmail.com

How to cite this article: Nivethaprashanthi S, Kavya R, Priyanga R, Vezhavendhan N. Comparing the Efficacy of Herbal and Non-herbal Toothpastes in Controlling Plaque and Gingivitis: A Review. J Sci Den 2020;10(1):25-27.

Source of support: Nil

Conflict of interest: None

To improve the efficiency of dentifrices in controlling the microorganisms, antimicrobials were added to it. ${ }^{6,7}$ Dentifrices serve as a day-to-day, convenient, and preventive tool available for good hygiene care of the oral cavity which helps in restricting the formation and growth of plaque, thereby preventing the oral diseases.

People move toward herbal toothpaste because of their view toward herbal products is much safer than non-herbal products that contain synthetic, chemical compounds. The adverse effects of non-herbal toothpaste are that it can contribute to ulcerations and lesions in the oral mucosa, perioral dermatitis, and so on. ${ }^{4}$

People interested toward herbal toothpaste have been increased recently. ${ }^{7}$ The main ingredients of the herbal toothpaste 
include herbs with historically established medicinal uses such as myrrh, which helps to destroy the microorganisms contributing to the formation of plaque; chamomile and sage, which decrease inflammation and bleeding of the gums; and Echinacea, which stimulates the body's defense mechanism. Essential oils are added to give flavor and relieve pain. Other ingredients include cloves, tea tree oil, coriander, lemon, and spearmint. ${ }^{8}$ This review is carried out to assess and compare the effectiveness of herbal and non-herbal toothpastes in controlling the dental plaque and gingivitis.

\section{Materials and Methods}

The following sources were searched from 2011 to 2019: PubMed and Google database. The following key words were used: herbal toothpaste, gingivitis, plaque, and non-herbal toothpaste. The primary focus of this study was to compare randomized control trials and clinical trials which used herbal toothpaste with non-herbal toothpaste in control and prevention of plaque and gingivitis. Clinical trials without randomization and other experimental studies were considered. The outcome measures were improvement in duration, objective and subjective difference in plaque accumulation, and gingivitis.

\section{Results}

The 13 articles that met the selection criteria (six randomized control trials and seven clinical trials) were included for this review in which plaque and gingival scores were recorded using Loe and Silness gingival index, Silness and Loe plaque index, OHI-S and plaque index (Turesky et al. modification of Quigley-Hein plaque index).

An average duration of follow-up ranges from 4 weeks to 24 weeks. Randomized control trial and nonrandomized control trial have shown significantly reduced plaque and gingival scores on an average duration on using herbal toothpaste. Studies (five randomized control trials and four clinical trials) state that there is a statistically equal reduction in plaque and gingival scores on using herbal and non-herbal toothpastes. The reduction in gingival and plaque scores was assessed by paired and unpaired $t$ test which shows no drastic significant change at baseline, on 3 months, and 6 months. No reports on serious reactions (bad taste and odor, hard to accept, and allergic reactions) on using herbal toothpaste.

\section{Discussion}

Maintaining a good oral hygiene prevents common oral diseases. The oral microbial flora contributes to most of the oral diseases and malodor. Thereby incorporating antimicrobial agents in the toothpaste can help the oral environment maintain a consistent phase. Dental plaque plays a major role in the etiology of caries, halitosis, gingivitis, and its progression to periodontitis. Mechanical management of plaque is considered as a best approach for eliminating it. A number of chemical plaque control agents have been studied, but on prolonged usage of these agents causes after effects. As a consequence, numerous herbal products appeared in the market, thereby promoting the benefits of them. The primary purpose of this study is to assess the effectiveness of herbal dentifrice in controlling dental plaque and gingivitis. ${ }^{9}$

Native herbal medicines are gaining special interest in the dental field for preventing and curing dental diseases. ${ }^{10}$ In controlling plaque and gingivitis, the herbal and non-herbal toothpastes are equally effective. Several studies stated that toothbrushing habit has significantly improved the hygiene status in both young and adult individuals. Dental caries, stains, and malodor can easily be prevented by brushing with toothpaste, and it can also enhance remineralization. ${ }^{8}$ Saxer et al. and Mullaly et al. found a significantly reduced plaque and gingival index score, but no significant difference was found between the herbal and non-herbal toothpastes. Willerhausen et al. stated in their study that the total salivary $\mathrm{pH}$ of the herbal product users has changed to be alkaline. ${ }^{6,10,11}$ George et al. stated that herbal toothpaste is effective in controlling plaque and gingivitis but has no statistically significant difference in the salivary $\mathrm{pH} 3$.

Ozaki et al. found herbal dentifrices to be as effective as the non-herbal ones in reduction of gingivitis. George et al., Mateu et al., and de Oliveira et al. found slightly lower efficacy of herbal products on gingivitis and gingival bleeding compared with conventional ones, whereas Sushma et al. and Pannuti et al. observed slightly higher gingivitis reduction with herbal products. de Oliveira et al. and Ozaki et al. found no adverse reactions to dentifrices products observed during the trial. ${ }^{9}$

Studies have concluded that herbal and non-herbal toothpastes are equally effective, and so people can conveniently use either of the toothpastes. ${ }^{711}$ Studies demonstrated reduced salivary glucose level after using herbal toothpaste which might be due to the presence of antidiabetic ingredients. Dant Kanti herbal toothpaste contains neem, babool, and Vajradanti that have properties of lowering the sugar. Himalaya complete care contains neem, pomegranate, and triphala which are antidiabetic substances. Vicco Vajradanti contains jamun, anantmul, amla, cinnamon, liquorice, and Vajradanti as antidiabetic ingredients. These substances are absorbed by the oral transmucosal route and are directly entered into the blood circulation which might further result in lowering the salivary glucose. ${ }^{12}$

Some studies showed that scaling and oral prophylaxis being carried out in the experimental phase, whereas in some it was not. However, there was no change in the efficacy of herbal and non-herbal toothpastes in controlling plaque and gingivitis. ${ }^{1-13}$ Toothbrush and paste usage promotes reduced plaque and gingival scores (Triratana et al. and Lindhe et al.). Clinical studies have proved herbal toothpastes are effective in reducing plaque and gingival scores demonstrating a significant plaque reduction from $7.17 \%$ to $61.2 \%$ and gingivitis reduction from $5.20 \%$ to $70.6 \%$. Herbal toothpastes are effective in reducing the plaque and gingivitis. Rubido et al. stated that medicated toothpaste significantly reduced bacterial count in the saliva. George et al. found no statistically significant difference between the herbal and conventional toothpastes on salivary $\mathrm{pH}$ values. Saxena et al. found in an in vitro study that herbal dentifrice showed the maximum inhibition zone. ${ }^{9}$

Some studies used herbal toothpaste (sudantha) containing Acacia chundra Wild, Adhatoda vasica Nees, Mimusops elengi L., seeds of Piper nigrum L., root of Pongamia pinnata L., Quercus infectoria Olivier., and Syzygium aromaticum L., Zingiber officinale Roscoe for improving oral hygiene and salivary microorganisms. ${ }^{13}$ Pongamia pinnata and Zingiber officinale Roscoe have antiinflammatory properties. For antimicrobial action, Terminalia chebula aralu and Quercus infectoria Oliv. are used. Syzygium aromaticum has an inhibitory effect toward periodontal pathogens (gram-negative, anaerobic) such as Prevotella intermedia and Porphyromonas gingivalis. Furthermore, Adhatoda vasica Nees reduces gingival bleeding, thereby improving inflammation of 
gingiva. Mimusops elengi is used in native medicine for curing gum diseases. ${ }^{4}$ Cinnamomum zeylanica is used as a flavoring agent which gives a good refreshing effect in the oral cavity. It has also been used for toothaches, malodor, and oral infections because of its antibacterial and antifungal properties. Dwivedi and Singh found that piperine has a significant antibiofilm effect on Streptococcus mutans. Anti-inflammatory property of Cinnamomum camphora helps in reducing swelling and pain associated with inflammation. ${ }^{10}$

Herbal toothpaste containing neem leaf extracts are effective in plaque reduction. Decrease in plaque scores may have been caused by the action of Azadirachta in neem leaf extracts, an antibacterial agent. Bhat et al. demonstrated that antibacterial solutions containing neem had the highest effectiveness in reducing the number of $S$. mutans when compared with turmeric, chlorhexidine, and cetylpyridinium chloride. ${ }^{2}$

A number of antiplaque agents such as chlorhexidine, delmopinol, hexetidine, stannous fluoride, triclosan, and other phenolic compounds have been incorporated in dental creams and evaluated for management of dental plaque (Cummins and Creeth, 1992; Baehni and Takeuchi, 2003). Synthetic toothpastes contain chemical substances that can produce harmful effects on prolonged usage (Mathias et al., 1980; Saxon et al., 1987; Maynard et al., 1993). Sodium lauryl sulfate is most widely used in detergent toothpaste that has been reported to cause adverse effects such as mucosal irritation and desquamation of oral soft tissues (Herlofson and Barkvoll, 1996). Ingredients like quaternary ammonium compound and chlorhexidine causes marked staining of teeth when used for prolonged duration of time. ${ }^{8}$

Cinnamon a component of herbal toothpaste can cause cinnamon contact stomatitis. A review was reported with case reports and series involving 35 cases secondary to the use of chewing gum with cinnamon flavor; after discontinuing the same, most of them recovered..$^{10} \mathrm{~A}$ number of studies have proved that herbal toothpastes do not cause adverse effects on the oral cavity and are equally effective in reducing plaque and gingivitis compared with fluoridated non-herbal dentifrice. Several studies have also proven the medicinal values of herbal products. Hence, medicated herbal toothpaste can be safely used to control plaque and gingivitis. ${ }^{5,9}$

\section{ConcLusion}

The conclusion of this review is that nowadays, there is an increase in the growth of interest on using herbal products among people. Herbal toothpastes are as effective as non-herbal toothpastes in controlling plaque and gingivitis. There is also no adverse reactions on using herbal toothpaste and can be used as an alternative to conventional (non-herbal) toothpaste. Henceforth, it depends on the preference of people to choose natural (herbal) or conventional (non-herbal) toothpaste.

\section{References}

1. Howshigan J, Perera K, Samita S, Rajapakse PS. The effects of an Ayurvedic medicinal toothpaste on clinical, microbiological and oral hygiene parameters in patients with chronic gingivitis: a double-blind, randomised, placebo-controlled, parallel allocation clinical trial. Ceylon Med J 2015;60(4):126-132. DOI: 10.4038/cmj.v60i4.8219.

2. Sugiarta AP, Lessang RN. Effect of herbal toothpaste containing neem leaves extract (azadirachta indica) against gingivitis: a clinical study. Int J Appl Pharm 2019;11(1):117-119. DOI: 10.22159/ijap.2019.v11s1. AR173.

3. He J, Deng Y, Zhu F, Zhong T, Luo N, Lei L, et al. The efficacy and safety of a herbal toothpaste in reducing gingivitis: a double-blind, randomized, placebo-controlled, parallel allocation clinical trial. Evid Based Complement Alternat Med 2019;2019:3764936. DOI: 10.1155/2019/3764936.

4. Gupta R, Ingle NA, Kaur N, Yadav P, Ingle E, Charania Z. Effectiveness of herbal and nonherbal fluoridated toothpaste on plaque and gingivitis: a randomized controlled trial. J Indian Assoc Public Health Dent 2015;13(3):218-221. DOI: 10.4103/2319-5932.165207.

5. Singh K, Singh P, Oberoi G. Comparative studies between herbal toothpaste (dantkanti) and nonherbal tooth paste. Int J Dent Res 2016;4(2):53-56. DOI: 10.14419/ijdr.v4i2.6633.

6. Hosadurga R, Boloor VA, Rao SN, MeghRani N. Effectiveness of two different herbal toothpaste formulations in the reduction of plaque and gingival inflammation in patients with established gingivitis-a randomized controlled trial. J Tradit Complement Med 2018;8(1):113119. DOI: 10.1016/j.jtcme.2017.04.005.

7. Ozaki F, Pannuti CM, Imbronito AV, Pessotti W, Saraiva L, Freitas NM, et al. Efficacy of a herbal toothpaste on patients with established gingivitis: a randomized controlled trial. Braz Oral Res 2006;20(2):172177. DOI: 10.1590/S1806-83242006000200015.

8. Hebbal M, Ankola AV, Sharma R, Johri S. Effectiveness of herbal and fluoridated toothpaste on plaque and gingival scores among residents of a working women's hostel-a randomised controlled trial. Oral Health Prev Dent 2012;10(4):389-395.

9. Tatikonda A, Debnath S, Chauhan VS, Chaurasia VR, Taranath M, Sharma AM. Effects of herbal and non-herbal toothpastes on plaque and gingivitis: a clinical comparative study. J Int Soc Prev Community Dent 2014;4(Suppl 2):S126-S129. DOI: 10.4103/2231-0762.146220.

10. Pentapati KC, Kukkamalla MA, Siddiq H, Sabnis N. Effectiveness of novel herbal dentifrice in control of plaque, gingivitis, and halitosisrandomized controlled trial. J Tradit Complement Med 2019. DOI: 10.1016/j.jtcme.2019.06.006.

11. George J, Hegde S, Rajesh KS, Kumar A. The efficacy of a herbalbased toothpaste in the control of plaque and gingivitis: a clinicobiochemical study. Indian J Dent Res 2009;20(4):480-482. DOI: 10.4103/0970-9290.59460.

12. Khairnar MR, Dodamani AS, Karibasappa GN, Naik RG, Deshmukh MA. Efficacy of herbal toothpastes on salivary $\mathrm{pH}$ and salivary glucose-a preliminary study. J Ayurveda Integr Med 2017;8(1):3-6. DOI: 10.1016/j. jaim.2016.12.004.

13. Jayashankar S, Panagoda GJ, Amaratunga EA, Perera K, Rajapakse PS. A randomised double-blind placebo-controlled study on the effects of a herbal toothpaste on gingival bleeding, oral hygiene and microbial variables. Ceylon Med J 2011;56(1):5-9. DOI: 10.4038/ cmj.v56i1.2887. 